\title{
Older Patients' Experiences of Sleep in the Hospital: Disruptions and Remedies
}

\author{
Chau Yuen Lee ${ }^{1}$, Lisa P.L. Low ${ }^{*}, 2$ and Sheila Twinn ${ }^{2}$ \\ ${ }^{1}$ Haven of Hope Hospital, Hong Kong \\ ${ }^{2}$ The Nethersole School of Nursing, The Chinese University of Hong Kong, Shatin, N.T., Hong Kong
}

\begin{abstract}
Complaints of disruptive sleep are common occurrences during hospitalization of older people, with unpleasant consequences arising when disrupted sleep is left unmanaged. This aim of this study was to explore the perceptions of Chinese older patients on the causes of sleep disturbance, sleeping pattern changes and sleep remedies that could be implemented to improve sleep quality during hospitalization. Sleep diaries and interviews highlighted that a crucial role of nurses is to listen and involve older patients into identifying their own sleep problems and sleep-promoting remedies they consider to work in their favour. Through understanding the sleep problems from patients' perspectives, nurses can appreciate the significance of disrupted sleep for the person concerned and effectively design needs-based sleep interventions to manage the changing sleep patterns and sleep needs of patients in the clinical area.
\end{abstract}

Keywords: Chinese, hospital, older patients, sleep.

\section{INTRODUCTION}

Sleep disturbance is a common phenomenon among older people. Complaints of disruptive sleep are not uncommon occurrences during hospitalisation, with unpleasant consequences arising when disrupted sleep is left unmanaged. In studies examining sleep experiences of patients in different care settings (medical, surgical, psychiatric and geriatric), one-half of them $(\mathrm{N}=438)$ experienced insufficient sleep and difficulty sleeping during the night [1]. Thus, complaints about disruptive sleep were likely to occur during hospitalization and were not entirely related to the clinical specialties patients were admitted into. This is rather alarming particularly when disrupted sleep, if prolonged, can lead to a chain of unpleasant consequences [2].

The causes of disruptive sleep can be regarded as interrelated and are categorized into physiological, environmental and psychological influences. Whilst physiological influences affecting sleep quality would include physical discomforts such as pain [1], these could worsen when patients are placed in noisy environments, where noise emanating from staff, patients and equipment has contributed to sleep loss [3]. This proceeds to patients having psychological concerns about the lack of quality sleep [4]. In terms of sleep disturbance arising as a result of disruption to usual personal lifestyle and habits, however, little has been mentioned about the patients' abilities to continue to perform their usual sleep routines in the hospital. According to Hodgson's study [2], the degree of sleep disruptions and its influence on a person's sleeping pattern is very personal and individualized. This suggests that if the priority is to meet the sleep needs of hospitalized patients, there should be an established procedure in place for nurses to readily ascertain the views of

*Address correspondence to this author at the Nethersole School of Nursing, The Chinese University of Hong Kong, Shatin, N.T., Hong Kong;

E-mail: lisalow@cuhk.edu.hk individual patients, whose sleep can be potentially influenced by the ward environment, such as in a thorough sleep assessment conducted at admission and thereafter. Gathering specific information about patients' perceptions of what causes disturbed sleep and strategies that would enable them to sleep better can provide nurses with in-depth understanding of the sleep needs and experiences of older hospitalized patients at and around the time of admission. Such knowledge can then be in-built into the nurses' day-to-day practice so as to develop and implement sleep strategies that will promote better sleep for their patients.

In dealing with sleep disruptions, a range of pharmacological and non pharmacological interventions has been recommended. To date, prescribed sedatives were more prominently used for hospitalized patients [5], despite no discernible differences were concluded regarding the sleep quality of patients using hypnotics compared with those who did not use them. However, $75 \%$ of these patients $(\mathrm{N}=32)$ taking hypnotics reported difficulties getting back to sleep after waking up at night-time [6]. Additionally, long-term use of sedatives has been ineffective in restoring sleep pattern changes [7].

More recently though, non pharmacological interventions have been considered as a better choice and the first line treatment for managing sleep disruptions among older people - an evolving consensus of physicians striving to do this in their practice [8]. Among the few studies exploring the impacts of non-pharmacological interventions on older people in nursing homes [9], and even less studies conducted on hospitalized elders [10], non-pharmacological interventions that are specific to nurses working on the wards can be classified into: those concerned with modifying the physical environment (e.g. lighting, noise, patient interruptions and daytime activities), and those that promoted relaxation and sleep (massage, music, guided imagery and progressive muscle relaxation) [10]. Moreover, in a computerized database 
search of interventions to facilitate adult sleep, twelve nonpharmacological interventions were identified to be within the scope of nursing practices. These nursing actions were measures that aimed to relax the sleeper, manage noise, repattern sleep-wake schedule, and inform sleepers about sleep hygiene [11]. Other non-pharmacological interventions that allowed the therapists to easily guide the therapy and would probably need additional training included: cognitive therapy, multi-component therapy and paradoxical intention [8]. Furthermore, the relaxing effects of aromatherapy and essential oils have been demonstrated to improve nighttime sleep of older residents in aged care facilities [12].

The promotion of non pharmacological sleep interventions among older patients seems a promising move forward, with the expectations of nurses to work closely with their patients to assess, review and meet individual sleep needs and practices $[6,11]$. In some instances, the use of a non pharmacological sleep protocol that had a combination of strategies (comprising a back rub, warm drink and relaxation tapes) was successfully conducted on a cohort of older patients in medical wards. The protocol provided a feasible, effective and non-toxic alternative to the use of sedativehypnotic drugs [14]. Despite the demonstrated efficacy of non-pharmacological interventions in promoting patients' sleep, many nurses remain cautious and feel these interventions were 'unrealistic', giving the existing demands of work in hospital settings [10]. Although the literature indicates the scope of non-pharmacological interventions for managing patients' sleep disturbance, little is actually known of what older patients perceive to be 'realistic' and 'effective' sleep interventions. More research is needed to include older patients' views on the impacts of hospitalization on their sleeping patterns and identify sleep strategies perceived by them to be useful and appropriate in improving sleep during a hospital stay.

Previous sleep studies have largely been conducted in acute care settings, with lesser attention given to examining patients' sleep pattern changes in extended care/rehabilitation settings. The limited literature available to inform sleep pattern changes of older patients during hospitalization and perceived helpful remedies to deal with disrupted sleep has prompted the need for this study. Therefore, the aim of this study was to examine Chinese older patients' experiences of sleep pattern changes, causes of disturbed sleep and the use of sleep remedies during a short hospital stay in an extended care ward in Hong Kong.

\section{MATERIALS AND METHODS}

A descriptive qualitative methodology was used on a convenience sample of six older male patients recruited from a Geriatric and Rehabilitation ward of a rehabilitation hospital. Following approval by the ethical committee, informed consent of eligible participants was obtained before collecting the data. Inclusion criteria were: participants had to stay

Table 1. Sleep Diaries of Participants During One Week of Hospitalization

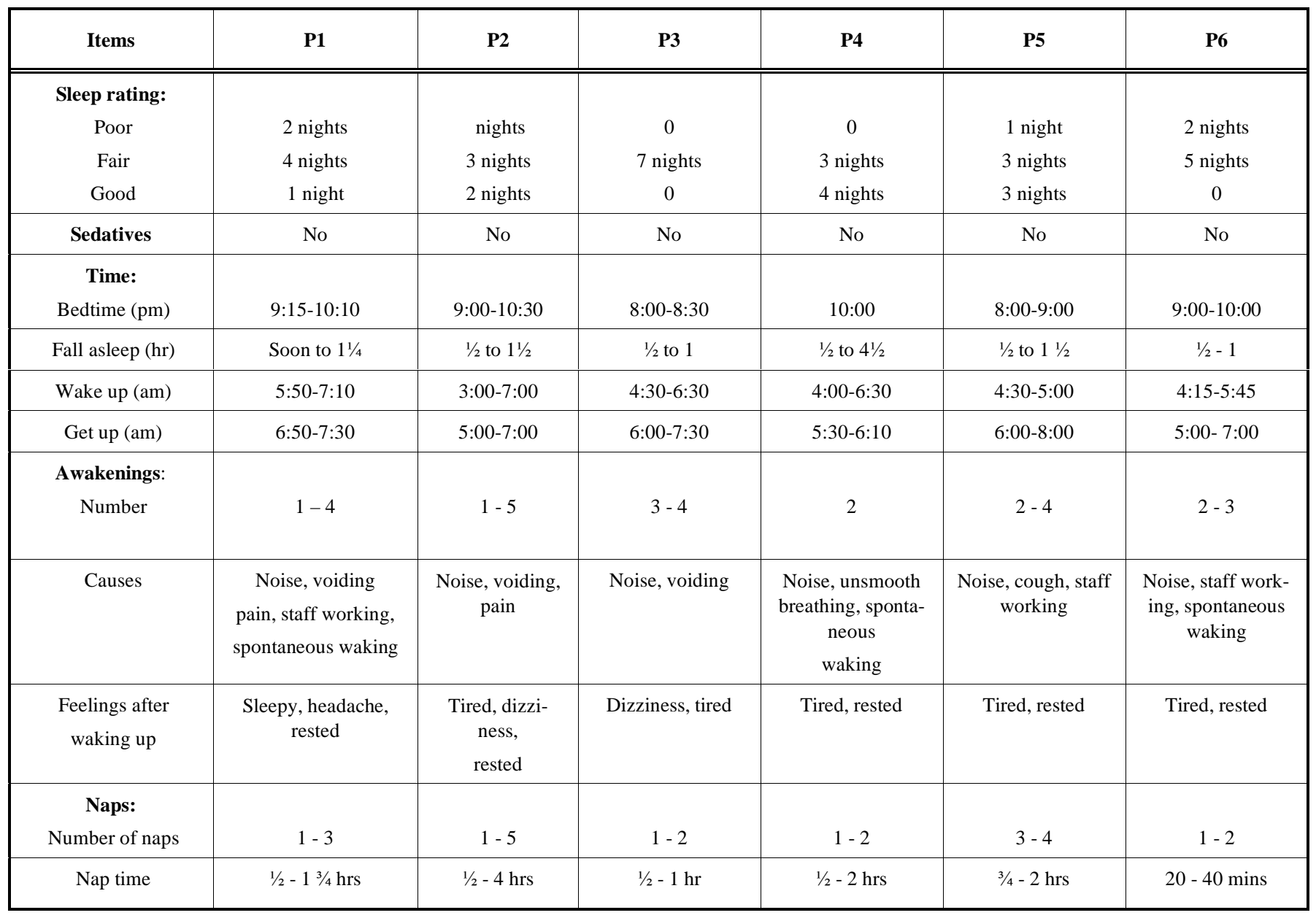


on the ward for three days to settle into the ward routine, spoke Cantonese, Chinese literate and cognitively intact. Participation was voluntary and participants' were informed of rights to withdraw from the study with no adverse effects on the care provided. Participants' identities were protected by using codes to ensure confidentiality.

In terms of data collection methods, self-completed sleep diaries (one sheet for each day over one week) and semistructured interviews were used to gather participants' personal sleep experiences during winter 2003 and spring 2004. The diary sheets (items used are found in Table 1) were completed from day four to ten after participants had woken and were collected on the same morning. The diary data were further explored and validated using audio-taped interviews so as to generate deeper understanding of older patients' sleep needs during the early days of hospitalization.

Steps taken to establish credibility of data included minimizing the role confusion of the researcher as data collector and her position as a nurse working in the research ward. This was achieved by not being on-duty in the hospital and not wearing uniform when approaching and interacting with the participants during data collection. Clarity of roles aided the establishment of a relationship that enabled participants to ask questions more freely during the collection of diary sheets, thereby reporting participants' perspectives as clearly and accurately as possible. Other steps involved pretesting the diary sheet to ensure that the format was simple and short to be completed by older patients. A delay in collecting diary data until day four of admission also allowed participants to attune to the ward routines and reported their experiences as accurately as they could. Lastly, the sleep diary data was also used to check the validity of the interview data. This paper presents the findings of the sleep diaries and two main interview issues pertaining to patients' perceived sleep disturbance and sleep-promoting remedies.

\section{RESULTS}

\section{Participants' Demographic Data}

Participants' age ranged from 65 to 79 years. Three were living with their family, two lived alone and one lived in an elderly home. Four participants completed primary education and two completed secondary education. All participants suffered from chronic health conditions including chronic obstructive airway disease, cerebral vascular accidents and hypertension.

\section{Patterns from Sleep Diaries}

Table 1 summarizes the sleep diaries of all participants during a one-week hospital stay. Sleeping patterns were fragmented, with most participants rating the quality of sleep to be fair. Most participants fell asleep in less than 90 minutes and night-time awakening did not exceed five times per night. Although no one required sedatives, about one half of participants napped more than twice per day. All participants agreed that noise was the main cause of night-time awakening, followed by voiding, staff working, waking spontaneously and being in pain. Although participants woke early, five participants recorded 'feeling rested' after waking, despite experiencing undesirable symptoms of sleepiness, headache, dizziness and tiredness. The analysis of the sleep diary data identified two major issues: patients' perceptions of sleep disturbance and helpful remedies to deal with disrupted sleep. These issues were also validated and illustrated by verbatim from the interview data.

\section{Patients' Perceptions of Sleep Disturbance}

Noise, pre-sleep thoughts and physical discomforts were the main causes of interrupted sleep. All participants described the 'sudden outburst' or 'loud breathing' of patients in critical or dependent conditions as persistently disturbing. The frequency of noise ranged from intermittent to all night long, and the volume was loud. Noise of staff was also problematical, with participants describing staff to be 'mindful of keeping their voices low and noise to a minimum when providing care to other patients during the night'. Discontentment arose when staff were engaged in 'unnecessary conversation about personal matters at night-time:

'Don't talk too much about personal matters at night when we're sleeping. It doesn't matter what they talk about in the daytime, but don't do so at night. It disturbs our sleep.' (C6)

Other causes of interrupted sleep were pre-sleep thoughts such as worrying about personal matters and unpleasant past memories. Once these thoughts were aroused, it was very difficult to stop them:

'Sometimes I worry too much. Sometimes it's my personal matters. I had some unhappy experiences. When I think of them, when I think of myself and what I have done, I'm unable to fall asleep. That's why I don't sleep well. I know I shouldn't think about it, but I can't stop myself. Sometimes it's like having a dream. The thoughts just come back after they've subsided. So it takes time for me to fall asleep.' (C6)

Physical discomfort such as nocturnal toileting, uncomfortable diapers, unrelieved muscular pain, feeling cold and unaccustomed to sleeping positions also provoked sleepless nights. A predominate concern of participants was the assurance that elimination needs were met before they could fall asleep. One patient described waking up with a sense of panic because of the need to urinate in an unfamiliar environment. Another patient described being kept awake because of wearing uncomfortable, bulky and wet diapers:

'During the first two nights, the nurses did not change my diapers often. It was not comfortable to be in a wet diaper, so I often couldn't fall asleep.' (C5)

\section{Helpful Sleep Remedies}

Sleep remedies were classified as two types: 'internal control' remedies were precisely cited to include exercising (e.g. body massage, Tai Chi, walking), listening to the radio, looking at the clock ticking, restricting fluids before bedtime, having positive thoughts, and requesting medication to relieve illness symptoms (e.g. shortness of breath or muscular pain). Comparatively, 'external control' remedies (those beyond participants' control) were less clearly defined. Predominately mentioned measures to improve the situation were described as 'beyond their personal control'. These included the aspiration for a quiet ward environment which could be achieved by 'moving noisy patients out of the ward' so as not to disturb the sleep of others, and 'nurses avoiding unnecessary night-time conversation'. Being able to have 
personal comforts met were also described as out of their control; for example, being offered favourite foods and drinks and 'making sure they were getting enough' so as to fall asleep easier. Although this finding was not examined in depth, further research could be conducted to examine patients' personal comfort and how nurses could be facilitated to meet patients' usual habits. Some participants would try to practice their usual pre-sleep rituals to get a sense of comfort and adapted their usual routine into the constraints of the ward.

Few participants spoke of bringing in lighter and warmer blankets and comfortable pillows similar to those used at home. About one half of participants were inclined to incorporate usual lifestyle preferences and habits (mainly nonpharmacological measures) into the ward routine. Examples included ensuring they got food or drinks they liked, and performing pre-sleep routines such as watching TV, reading a book, or brushing their teeth.

\section{DISCUSSION}

This study used sleep diaries and interviews at around the time of admission to detect the changing sleep needs of patients, although this paper predominately reported findings arising from the sleep diaries. Findings highlighted that patients' sleep needs were best addressed by enabling them to identify their sleep problems and sleep-promoting remedies they believe will work. Only through an understanding of the sleep problems from the patients' perspectives can nurses begin to appreciate the significance of disrupted sleep for the person concerned and thus effectively design needs-based sleep interventions to manage the changing sleep patterns and sleep needs of patients in the clinical area.

Congruent with results from acute care settings [3, 4], noise was a major perception of sleep disturbance in this extended care ward. Through listening to older patients, marked individual differences in sensitivity and tolerance to noise levels were revealed. Although most people would rapidly (within a few days) adapt to sleeping in a noisy environment, adaptation was never completely achieved [15]. Others have found that people who were familiar with the noise can become accustomed to constant noise after time and might not be easily awakened by it [16]. However, the sudden and infrequent noise from this study appeared to cause more disruptive sleep to older patients. Another point worth noting was that nurses were less aware and oblivious to the inappropriate noise arising from their own behaviour, such as social chatting. Interestingly, no one mentioned that noisy equipment caused disrupted sleep, probably as extended care wards mainly catered for long-stay patients with non-acute conditions. Since the study did not record the frequency of nocturnal awakening due to voiding, it is possible to underestimate the incidence and impact of nocturnal voiding, particularly as patients only reported when situations became unbearable [17], and deliberately restricting fluids to 'normalize' and 'accept' nocturia symptoms [18]. Detecting sleep problems would therefore be challenging for staff unless practices were in place to ascertain patients' usual sleep patterns and sleep needs at different times during hospitalisation. In this way, in-depth assessment of other aspects of the patients' lives that impact on sleep can be considered.
The findings of this study also highlighted the importance of making careful assessment when using diapers to meet older patients' elimination needs. Studies have shown that this group of clients slept less well and were either usually kept awake because of the wetness or interrupted by nurses performing hygiene care [19]. This practice was shown to hamper some participants' independence with elimination, as one patient did not use diapers at home and was able to eliminate independently. Justifying its use and monitoring patients' comfort will prevent this practice from being regarded as an 'easy and convenient way' of toileting patients.

Regarding interrupted thoughts, although these thoughts are known to produce either pleasant, unpleasant or emotionally neutral effects, such thoughts were intrusive, difficult to stop and engaged people in more cognitive activities once started [15]. Pre-sleep thoughts among patients were noted to provoke disruptive nights. Recognizing the impacts of presleep thoughts on the patient's usual sleep pattern would enable nurses to initiate prompt interventions and provide appropriate counseling to prevent further sleep deterioration. Although pre-sleep Pre-sleep thoughts can either produce pleasant, unpleasant or emotionally neutral effects, such thoughts were intrusive, difficult to stop and engage the thinkers in more cognitive activities [15]. It does, however, mean that nurses would need to go beyond the usual initial assessment of sleep and rest, and to include in-depth assessments of other aspects of the patients' lives that could impact on sleep.

Since no participants used sedatives, they were more inclined to suggest the use of different kinds of nonpharmacological sleep remedies to deal with sleep disturbances. In the ward situation, remedies were not complex, expensive or required high level nursing care. On the contrary, they were simple, accessible and useful techniques for overcoming sleep disruptions. This study therefore suggests a scope for integrating non-pharmacological sleep interventions into the daily work of nurses in the hospital. However, patients' individual preferences and usual habits should be addressed in the promotion of these interventions. For example, contradictory to previous findings suggesting that staring at the clock heightened the state of arousal in patients, who then worry about the time remaining for sleep [20], this study found that it was easier to fall asleep whilst looking at the clock. Such difference highlights the importance of considering individual preferences and usual sleep habits when proposing sleep approaches to meet patients sleep needs. Whilst all participants agreed a quiet ward environment was crucial to a good night's sleep, staff were responsible for 'taking suitable actions' in ensuring this would happen. Given that this was an unlikely occurrence, the importance of 'adapting' to the noisy and unfamiliar ward was supported in the sleep diaries, which illustrated participants becoming 'gradually accustomed' to the noise, staff working, and sleeping in the presence of unfamiliar people.

Since a longer period of adjustment is required for older patients to adapt to sleeping environment of hospitals, creating a conducive sleep environment is vital in promoting the health and well-being of our older patients. Understanding patients' sleep problems will allow nurses to appreciate the significance of disrupted sleep and effectively design needs- 
based sleep interventions for the person concerned. Since older patients needed longer time to adapt to sleeping in hospital environments, further studies to examine how remedies proposed can be integrated into the ward routine and improve sleep is suggested.

In terms of the methodology, the findings suggested that sleep diaries provided a practical method of enabling patients to identify their sleep needs around the time of admission, known to be a stressful time filled with unknown expectations. The baseline information collected on sleep/wake patterns serve to substantiate the sleep assessment data collected during admission. This approach actively involves patients in their care and regularly reviews sleep needs at different times during hospitalization. Such knowledge can be in-built into the daily ward practice and develop sleep strategies to improve patients' sleep.

\section{CONCLUSIONS}

In this study, sleep diaries and sleep interviews were used around the time of admission to detect the changing sleep needs of Chinese older hospitalized patients. The findings highlighted that patients' sleep needs were best addressed by enabling them to assess and identify their sleep problems and sleep-promoting remedies they believe will work. This information could be collected at different times during a hospital stay, with the aim to identify the sleep needs and (potential) difficulties of patients as soon as possible following admission [6]. However, these assessments seems to place greater emphasis on the abilities of nurses to conduct accurate and thorough sleep assessment, rather than involving the patients in the process of identifying their own sleep needs and sleep remedies. Understanding patients' sleep problems will allow nurses to appreciate the significance of disrupted sleep and effectively design needs-based sleep interventions for the person concerned. Since older patients needed longer time to sleep in the hospital environments, further studies to examine how remedies proposed can be integrated into the ward routine and improve sleep is suggested. The study therefore suggests a scope for promoting and integrating non-pharmacological sleep interventions into the daily work of nurses in the hospital. However, conducting a larger study to elicit older patients' perspectives on how non pharmacological sleep measures minimised disturbed sleep is suggested so as to better equip nurses at the ward level with knowledge on how to proceed with this endeavor.

\section{REFERENCES}

[1] Southwell MT, Wistow G. Sleep in hospitals at night: are patients' needs being met. J Adv Nurs 1995; 21: 1101-09.

[2] Hodgson LA. Why do we need sleep? Relating theory to nursing practice. J Adv Nurs 1991; 16: 1503-10.

[3] Freedman NS, Kotzer N, Schwab RJ. Patient perception of sleep quality and etiology of sleep disruption in the Intensive Care Unit. Am J Respir Crit Care Med 1999; 159: 1155-62.

[4] Tranmer JE, Minard J, Fox LA, Rebelo L. The sleep experience of medical and surgical patients. Clin Nurs Res 2003; 12: 153-73.

[5] O'Reilly R, Rusnak C. The use of sedative hypnotic drugs in a university teaching hospital. CMAJ 2000; 142: 585-89.

[6] Ersser S, Wiles A, Taylor H, Wade S, Walsh R, Bentley T. The sleep of older people in hospital and nursing homes. J Clin Nurs 1999; 8: 360-68.

[7] Glass J, Lanctot KL, Herrmann N, Sproule BA, Busto UE. Sedative hypnotics in older people with insomnia: meta-analysis of risks and benefits. BMJ 2005; 331: 1169-75.

[8] Petit L, Azad N, Byszewski A, Sarazan F, Power B. Nonpharmacological management of primary and secondary insomnia among older people: review of assessment tools and treatments. Age Ageing 2003; 32: 19-25.

[9] Alessi CA, Martain JL, Webber AP, Kim EC, Harker JO, Josephson KR. Randomized, controlled trial of a nonpharmacological intervention to improve abnormal sleep/wake patterns in nursing home residents. J Am Geriatr Soc 2005; 53: 80310 .

[10] Nagel CL, Markie MB, Richards KC, Taylor JL, Beverly C. Sleep promotion in hospitalized elders. Medsurg Nurs 2005; 12: 279-92.

[11] Floyd JA, Falahee ML, Fhobir RH. Creation and analysis of a computerized database of interventions to facilitate adult sleep. Nurs Res 2000; 49: 236-41.

[12] Joanna Briggs Institute for Evidence Based Nursing and Midwifery. Best practice. Strategies to manage sleep in residents of aged care facilities Evidence Based Practice Information Sheets for Health Professionals; 2004; 8: 1-6.

[13] Foreman M, Wykle M. Nursing standard of practice protocol: sleep disturbances in elderly patients. Geri Nurs 1995; 16: 238-43.

[14] McDowell JA, Mion LC, Lydon TJ, Inouye SK. A nonpharmacologic sleep protocol for hospitalized older patients. J Am Geriatr Soc 1998; 46: 700-05.

[15] Morgan K, Closs SJ. Sleep management in nursing practice: an evidence-based guide. London: Churchill Livingstone; 1999.

[16] Douglas N J. Clinicians' guide to sleep medicine. New York: Oxford University Press; 2002.

[17] Umlanf MG, Goode S, Burgio KL. Psychosocial issues in geriatric urology: problems in treatment and treatment seeking. Urol Clin North Am 1996; 23: 127-136.

[18] Donahue JL, Lowenthal DT. Nocturnal polyuria in the elderly person. Am J Med Sci 1997; 314: 232-38

[19] Hoeman SP. Rehabilitation nursing: process, application, and outcomes. 3rd ed. St Louis: Mosby; 2002.

[20] Vincent J, Zacrone JR. Sleep hygiene. In: Kryger MH, Dement WC. Principles and practices of sleep medicine. 3rd ed. New York: Saunders; 2000, pp. 657-75. 